\title{
Streptomyces stramineus sp. nov., a New Species of the Verticillate Streptomycetes
}

\author{
D. P. LABEDA, ${ }^{1 *}$ M. P. LECHEVALIER, ${ }^{2}$ AND R. T. TESTA ${ }^{3}$ \\ Microbial Properties Research, National Center for Agricultural Utilization Research, Agricultural Research Service, \\ U.S. Department of Agriculture, Peoria, Illinois $61604^{1}$; R.R. 2, Box 2235, Morrisville, Vermont 05661'; \\ and Infectious Disease Section, Wyeth-Ayerst Research, Pearl River, New York $10965^{3}$
}

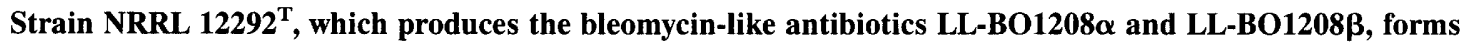
umbels consisting of chains of smooth-surface ovoid spores that are borne on verticils on the aerial mycelia, indicating that it is a member of the verticillate group of the genus Streptomyces formerly classified in the genus Streptoverticillium. This strain was compared morphologically and physiologically to 54 other verticillate Streptomyces strains. The levels of DNA relatedness between strain NRRL $12292^{\mathrm{T}}$ and 34 other verticillate Streptomyces strains, including strains representing at least 19 genetic species clusters, were also determined. Strain NRRL $12292^{\mathrm{T}}$ is morphologically and physiologically distinct from the other verticillate strains studied, particularly because of the straw yellow color of its aerial mycelia and spore mass. DNA hybridization data support the uniqueness of this strain, since the levels of DNA relatedness between NRRL ${ }^{2292}{ }^{\mathrm{T}}$ and the other verticillate strains used in this study were low. Our data support designation of a new species, Streptomyces stramineus, whose type strain is NRRL 12292.
\end{abstract}

Streptomyces sp. strain NRRL $12292^{\mathrm{T}}$ was isolated from a soil sample from South Dakota during the course of a naturalproducts screening program at Lederle Laboratories. This strain produces the antibiotics LL-BO1208 $\alpha$ and LL-BO1208 $\beta$, which are related to phleomycin. Morphological observations of the strain revealed that it produced spores from verticils on the aerial mycelium, which is typical of microorganisms previously described as members of the genus Streptoverticillium. The gross colonial morphology of strain NRRL $12292^{\mathrm{T}}$ was distinctive when it was compared to the gross colonial morphology of other verticillate streptomycetes because of the straw yellow color of the aerial mass. The genus Streptoverticillium has been reunited with the genus Streptomyces based on $16 S$ rRNA sequence similarity data (17). Strain NRRL $12292^{\mathrm{T}}$ was therefore compared phenotypically with other verticillate streptomycetes formerly classified in the genus Streptoverticillium and was found to be a member of a new species. Subsequently, we determined levels of DNA relatedness spectrophotometrically between NRRL $12292^{\mathrm{T}}$ and 34 representative strains belonging to 19 species of verticillate streptomycetes previously delineated (5) based on levels of DNA relatedness greater than $70 \%$.

\section{MATERIALS AND METHODS}

Strains and cultivation conditions. The strains used in this study are listed in Table 1. Primary storage of strains was in refrigerated ampoules containing freeze-dried spore and vegetative mycelium suspensions in sterile beef serum. Working stock cultures were maintained on slants of synthetic starch agar or yeast extract-malt extract agar $(15)$ and were stored at $4^{\circ} \mathrm{C}$ until they were needed. The inoculum used for physiological evaluation was grown in Bennett's broth ( $1 \mathrm{~g}$ of yeast extract per liter, $1 \mathrm{~g}$ of beef extract per liter, $2 \mathrm{~g}$ of NZamine type A per liter, $10 \mathrm{~g}$ of glucose per liter; $\mathrm{pH}$ 7.4). The biomass used for extraction of DNA was grown in tryptone-glucose-liver extract-yeast extract broth (12) with shaking at $200 \mathrm{rpm}$ at $28^{\circ} \mathrm{C}$.

Morphological observations. Gross morphological observations were made by using cultures grown for 14 days at $28^{\circ} \mathrm{C}$ on the standard media suggested by the International Streptomyces Project (ISP) (15). Micromorphology and sporulation were determined by light microscopy and scanning electron microscopy. The samples used for scanning electron microscopy were samples from 14-day cul-

* Corresponding author. Mailing address: National Center for Agricultural Utilization Research, 1815 North University Street, Peoria, IL 61604 tures on agar media that had been fixed overnight with osmium tetroxide vapors, postfixed for $1 \mathrm{~h}$ in $1 \%$ osmium tetroxide in Millonig's phosphate-buffered fixative (10), dehydrated through a graded acetone series, critical point dried from liquid $\mathrm{CO}_{2}$, and sputter coated with gold-palladium. The samples were observed with a JEOL model JSM $6400 \mathrm{~V}$ scanning electron microscope.

Chemotaxonomic methods. Diaminopimelic acid analysis was carried out by using the procedures of Becker et al. (1), as modified by Staneck and Roberts (16). The fatty acid content was determined by using biomass collected from Trypticase soy broth (BBL, Cockeysville, Md.) agar plates that had been incubated for 4 days at $28^{\circ} \mathrm{C}$. Growth scraped from the plates was processed immediately or frozen at $-20^{\circ} \mathrm{C}$ until it was processed. Fatty acid methyl esters were obtained from the biomass by saponification, methylation, and extraction by using the method of Miller and Berger (9). The fatty acid methyl esters were separated and quantified by using a model $9898 \mathrm{~A}$ gas chromatograph (HewlettPackard, Inc., Avondale, Pa.) fitted with a methyl phenyl silicone fused silica capillary column ( $25 \mathrm{~m}$ by $0.2 \mathrm{~mm}$; Hewlett-Packard) and Microbial Identification System software (MIDI, Inc., Newark, Del.). Peaks were automatically integrated and fatty acid identities and percentages were determined by the Microbial Identification System software.

Physiological tests. Physiological tests, including tests to determine production of acid from carbohydrates, utilization of organic acids, growth in the presence of glycerol, lysozyme, salicylate, and $5 \% \mathrm{NaCl}$, and hydrolysis and decomposition of adenine, guanine, hypoxanthine, tyrosine, xanthine, casein, esculin, urea, and nitrate, were performed by using the media of Gordon et al. (2). Phosphatase activity was evaluated by using the method of Kurup and Schmitt (4). The tests to determine utilization of carbohydrates as the sole carbon source for growth were performed with the media of Pridham and Gottlieb (11).

DNA isolation, purification, and hybridization. DNA was extracted from cells by the method of Marmur ( 7 ) after the cells were broken by passing them through a French pressure cell (SLM Instruments, Inc., Urbana, Ill.) at 10,000 $\mathrm{lb} / \mathrm{in}^{2}$. The DNA was further purified by chromatography on hydroxyapatite or by cesium chloride density gradient centrifugation (6). The guanine-plus-cytosine $(\mathrm{G}+\mathrm{C})$ content of the DNA was determined spectrophotometrically from the midpoint of thermal denaturation in $1 \times$ SSC $(0.15 \mathrm{M} \mathrm{NaCl}$ plus $0.015 \mathrm{M}$ sodium citrate) (8). The levels of DNA relatedness between strains were calculated from $\mathrm{C}_{0} t_{0.5}$ values in $5 \times \mathrm{SSC}$ supplemented with $20 \%$ dimethyl sulfoxide at $65^{\circ} \mathrm{C}\left(T_{m}-\right.$ $-23^{\circ} \mathrm{C}$ ) by the method of Seidler et al. (13) and Seidler and Mandel (14) as described by Kurtzman et al. (3).

Statistical calculations. Statistical calculations on the DNA relatedness data were performed by using SAS for Windows, release 6.11 (SAS Institute, Inc., Cary, N.C.), and a FoxTech Guardian 150-MHz Pentium microcomputer (Fox Technologies, Inc., Yorkville, Ill.). The data were clustered by the average linkage algorithm with the SAS/STAT CLUSTER procedure, and a dendrogram was generated with SAS/GRAPH by using the macro GRAFTREE written and kindly provided by Dan Jacobs, University of Maryland.

\section{RESULTS}

Morphological and chemotaxonomic properties. The verticillate sporophores on the aerial mycelia of Streptomyces sp. 
TABLE 1. Streptomyces strains used in this study

\begin{tabular}{|c|c|c|}
\hline Taxon & Strain & Characteristic(s) examined $^{a}$ \\
\hline S. abikoensis & NRRL B-2113 ${ }^{\mathrm{T}}\left(=\right.$ ATCC $\left.12766^{\mathrm{T}}\right)$ & DNA, PHYS \\
\hline S. ardus & NRRL $2817^{\mathrm{T}}\left(=\right.$ ATCC $\left.27417^{\mathrm{T}}\right)$ & DNA, PHYS \\
\hline$S$. albireticuli & NRRL B-5493 ${ }^{\mathrm{T}}\left(=\right.$ ATCC $\left.19721^{\mathrm{T}}\right)$ & PHYS \\
\hline S. aspergilloides & NRRL B-3355 $\left(=\right.$ ATCC $\left.14808^{\mathrm{T}}\right)$ & PHYS \\
\hline S. aureoversilis & NRRL B-3325 ${ }^{\mathrm{T}}\left(=\right.$ ATCC $\left.15853^{\mathrm{T}}\right)$ & DNA, PHYS \\
\hline S. baldaccii & NRRL B-3500 ${ }^{\mathrm{T}}\left(=\right.$ ATCC $\left.23654^{\mathrm{T}}\right)$ & DNA, PHYS \\
\hline S. biverticillatus & NRRL ISP-5272 $2^{\mathrm{T}}\left(=\right.$ ATCC $\left.23615^{\mathrm{T}}\right)$ & DNA, PHYS \\
\hline S. blastmyceticus & NRRL B-5480 ${ }^{\mathrm{T}}\left(=\right.$ ATCC $\left.19731^{\mathrm{T}}\right)$ & DNA, PHYS \\
\hline S. caespitosus & NRRL $2564^{\mathrm{T}}\left(=\right.$ ATCC $\left.27422^{\mathrm{T}}\right)$ & PHYS \\
\hline S. cinnamoneus subsp. albosporeus & NRRL B-5624 $\left(=\right.$ ATCC $\left.25186^{\mathrm{T}}\right)$ & DNA, PHYS \\
\hline S. cinnamoneus subsp. azacoluta & NRRL B-1699 ${ }^{\mathrm{T}}\left(=\right.$ ATCC $\left.12686^{\mathrm{T}}\right)$ & DNA, PHYS \\
\hline S. cinnamoneus subsp. cinnamoneus & NRRL B-1285 $\left(=\right.$ ATCC $\left.11874^{\mathrm{T}}\right)$ & DNA, PHYS \\
\hline S. cinnamoneus subsp. lanosus & ATCC $25187^{\mathrm{T}}$ & PHYS \\
\hline S. cinnamoneus subsp. sparsus & ATCC $25185^{\mathrm{T}}$ & PHYS \\
\hline S. distallicus & NRRL $2886^{\mathrm{T}}\left(=\mathrm{DSM} 40846^{\mathrm{T}}\right)$ & DNA, PHYS \\
\hline S. ehimensis & NRRL B-1967 $\left(=\right.$ ATCC $\left.23903^{\mathrm{T}}\right)$ & PHYS \\
\hline S. eurocidicus & NRRL B-1676 ${ }^{\mathrm{T}}\left(=\right.$ ATCC $\left.27428^{\mathrm{T}}\right)$ & DNA, PHYS \\
\hline S. fervens & NRRL $2755^{\mathrm{T}}\left(=\right.$ ATCC $\left.27429^{\mathrm{T}}\right)$ & DNA, PHYS \\
\hline S. flavopersicus & NRRL $2820^{\mathrm{T}}\left(=\right.$ ATCC $\left.19756^{\mathrm{T}}\right)$ & DNA, PHYS \\
\hline S. griseocarneus & NRRL B-1068 ${ }^{\mathrm{T}}\left(=\right.$ ATCC $\left.12628^{\mathrm{T}}\right)$ & DNA, PHYS \\
\hline S. griseoverticillatus & NRRL B-12432 $2^{\mathrm{T}}\left(=\right.$ ATCC $\left.27436^{\mathrm{T}}\right)$ & DNA, PHYS \\
\hline S. hachijoensis & NRRL B-3106 $6^{\mathrm{T}}\left(=\text { ATCC } 19769^{\mathrm{T}}\right)^{\prime}$ & DNA, PHYS \\
\hline S. hiroshimensis & NRRL B-5484 ${ }^{\mathrm{T}}\left(=\right.$ ATCC $\left.19722^{\mathrm{T}}\right)$ & DNA, PHYS \\
\hline S. kashmirensis & NRRL B-3103 ${ }^{\mathrm{T}}\left(=\right.$ ATCC $\left.27439^{\mathrm{T}}\right)$ & PHYS \\
\hline S. kentuckensis & NRRL B-1831 ${ }^{\mathrm{T}}\left(=\right.$ ATCC $\left.12691^{\mathrm{T}}\right)$ & DNA, PHYS \\
\hline S. kishiwadensis & NRRL B-12326 ${ }^{\mathrm{T}}\left(=\right.$ ATCC $\left.25464^{\mathrm{T}}\right)$ & PHYS \\
\hline S. ladakanum & NRRL $3191^{\mathrm{T}}\left(=\right.$ ATCC $\left.27441^{\mathrm{T}}\right)$ & DNA, PHYS \\
\hline S. lavenduligriseus & NRRL B-3173 $\left(=\right.$ ATCC $\left.13306^{\mathrm{T}}\right)$ & DNA, PHYS \\
\hline S. lilacinus & NRRL B-1968 ${ }^{\mathrm{T}}\left(=\right.$ ATCC $\left.23930^{\mathrm{T}}\right)$ & PHYS \\
\hline "S. luteoreticuli" & NRRL B-12435 (= ATCC 27446) & DNA, PHYS \\
\hline S. luteosporeus & NRRL $2401^{\mathrm{T}}\left(=\right.$ ATCC $\left.33049^{\mathrm{T}}\right)$ & PHYS \\
\hline S. luteoverticillatus & NRRL B-1995 ${ }^{\mathrm{T}}\left(=\right.$ ATCC $\left.23933^{\mathrm{T}}\right)$ & PHYS \\
\hline S. mashuensis & NRRL B-8164 ${ }^{\mathrm{T}}\left(=\right.$ ATCC $\left.23934^{\mathrm{T}}\right)$ & DNA, PHYS \\
\hline S. mobaraensis & NRRL B-3729 $\left(=\right.$ ATCC $\left.29032^{\mathrm{T}}\right)$ & DNA, PHYS \\
\hline S. morookaense & NRRL B-12429 ${ }^{\mathrm{T}}\left(=\right.$ ATCC $\left.19166^{\mathrm{T}}\right)$ & PHYS \\
\hline S. netropsis & NRRL $2268^{\mathrm{T}}\left(=\right.$ ATCC $\left.23940^{\mathrm{T}}\right)$ & DNA, PHYS \\
\hline S. olivoreticuli & NRRL B-2091 ${ }^{\mathrm{T}}\left(=\right.$ ATCC $\left.23943^{\mathrm{T}}\right)$ & PHYS \\
\hline S. olivoverticillatus & NRRL B-1994 ${ }^{\mathrm{T}}\left(=\right.$ ATCC $\left.25480^{\mathrm{T}}\right)$ & DNA, PHYS \\
\hline S. orinoci & NRRL B $3379^{\mathrm{T}}\left(=\right.$ ATCC $\left.23202^{\mathrm{T}}\right)$ & PHYS \\
\hline S. parvisporogenes & NRRL B-5464 ${ }^{\mathrm{T}}\left(=\right.$ ATCC $\left.12568^{\mathrm{T}}\right)$ & PHYS \\
\hline "S. pentaticus subsp. jenensis" & NRRL B-12088 (= DSM 40848) & DNA, PHYS \\
\hline "S. reticulusruber" & NRRL B-1484 (= ATCC 25607) & DNA, PHYS \\
\hline S. roseoverticillatus & NRRL B-1993' $\left(=\right.$ ATCC $\left.19807^{\mathrm{T}}\right)$ & DNA, PHYS \\
\hline S. roseoverticillatus subsp. albosporus & ATCC $25189^{\mathrm{T}}$ & PHYS \\
\hline "S. rubroverticillatus" & NRRL B-16433 (= DSM 41489) & DNA \\
\hline S. salmonis & NRRL B-1472 ${ }^{\mathrm{T}}\left(=\right.$ DSM $\left.40895^{\mathrm{T}}\right)$ & DNA \\
\hline S. septatus & NRRL $2974^{\mathrm{T}}\left(=\right.$ ATCC $\left.27464^{\mathrm{T}}\right)$ & PHYS \\
\hline S. sporiferus & ATCC $25188^{\mathrm{T}}$ & PHYS \\
\hline "S. taitoense" & NRRL B-16435 (= ATCC 29758) & DNA, PHYS \\
\hline "S. takataensis" & NRRL B-12516 (= ATCC 27469) & DNA, PHYS \\
\hline "S. tropicalensis" & NRRL B-12481 (= ATCC 17963) & DNA, PHYS \\
\hline S. thioluteus & ATCC $12310^{\mathrm{T}}$ & PHYS \\
\hline S. waksmanii & NRRL B-1707 $\left(=\right.$ ATCC $\left.12629^{\mathrm{T}}\right)$ & DNA, PHYS \\
\hline
\end{tabular}

${ }^{a}$ DNA, level of DNA relatedness; PHYS, physiological profile.

strain NRRL $12292^{\mathrm{T}}$ were observed on many media by light microscopy, as shown in Fig. 1, and were also clearly visible on a scanning electron micrograph (Fig. 2). Each of the verticils produced umbels consisting of straight chains of ovoid smooth- surface spores $(0.5$ by $1.0 \mu \mathrm{m})$. This morphology is typical of strains previously assigned to the genus Streptoverticillium.

The gross colonial morphology of a 14-day growth of strain NRRL $12292^{\mathrm{T}}$ on ISP media is shown in Table 2. The straw

TABLE 2. Morphological characteristics of S. stramineus NRRL $12292^{\mathrm{T}}$

\begin{tabular}{|c|c|c|c|c|}
\hline Medium & Growth & $\begin{array}{l}\text { Aerial mycelium-spore } \\
\text { mass color }\end{array}$ & $\begin{array}{l}\text { Vegetative my- } \\
\text { celium color }\end{array}$ & $\begin{array}{l}\text { Soluble } \\
\text { pigments }\end{array}$ \\
\hline Yeast extract-malt extract agar (ISP medium 2) & Good & Yellow & Tan & Light brown \\
\hline Inorganic salts-soluble starch agar (ISP medium 4) & Good & Yellow & Yellow-white & None \\
\hline Glycerol-asparagine agar (ISP medium 5) & Good & Yellow & Yellow & None \\
\hline Oatmeal agar (ISP medium 3) & Good & Yellow & Yellow & None \\
\hline
\end{tabular}




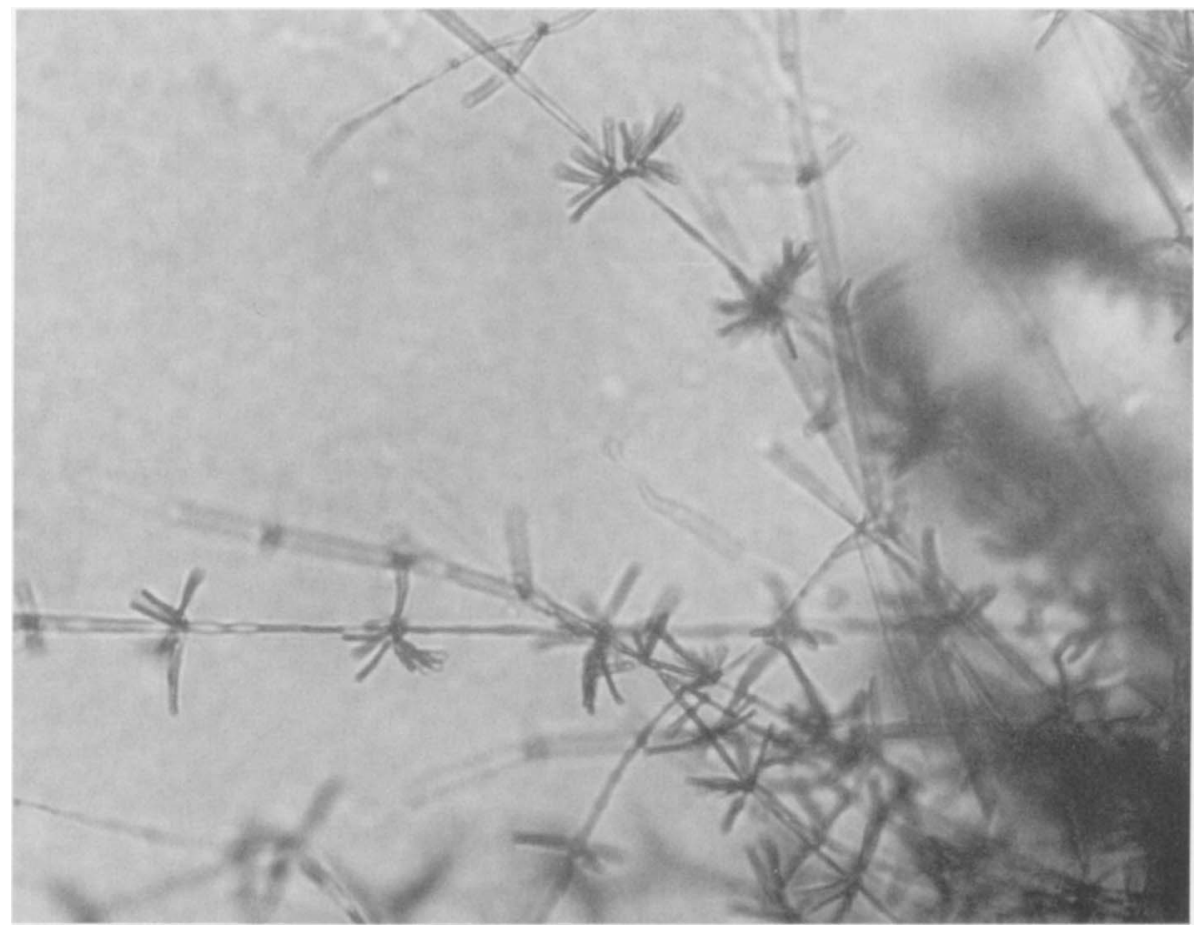

FIG. 1. Light micrograph of a 24-day culture of $S$. stramineus NRRL $12292^{\mathrm{T}}$ on Czapek agar. Note the umbels of spores borne on verticils on the aerial hyphae.

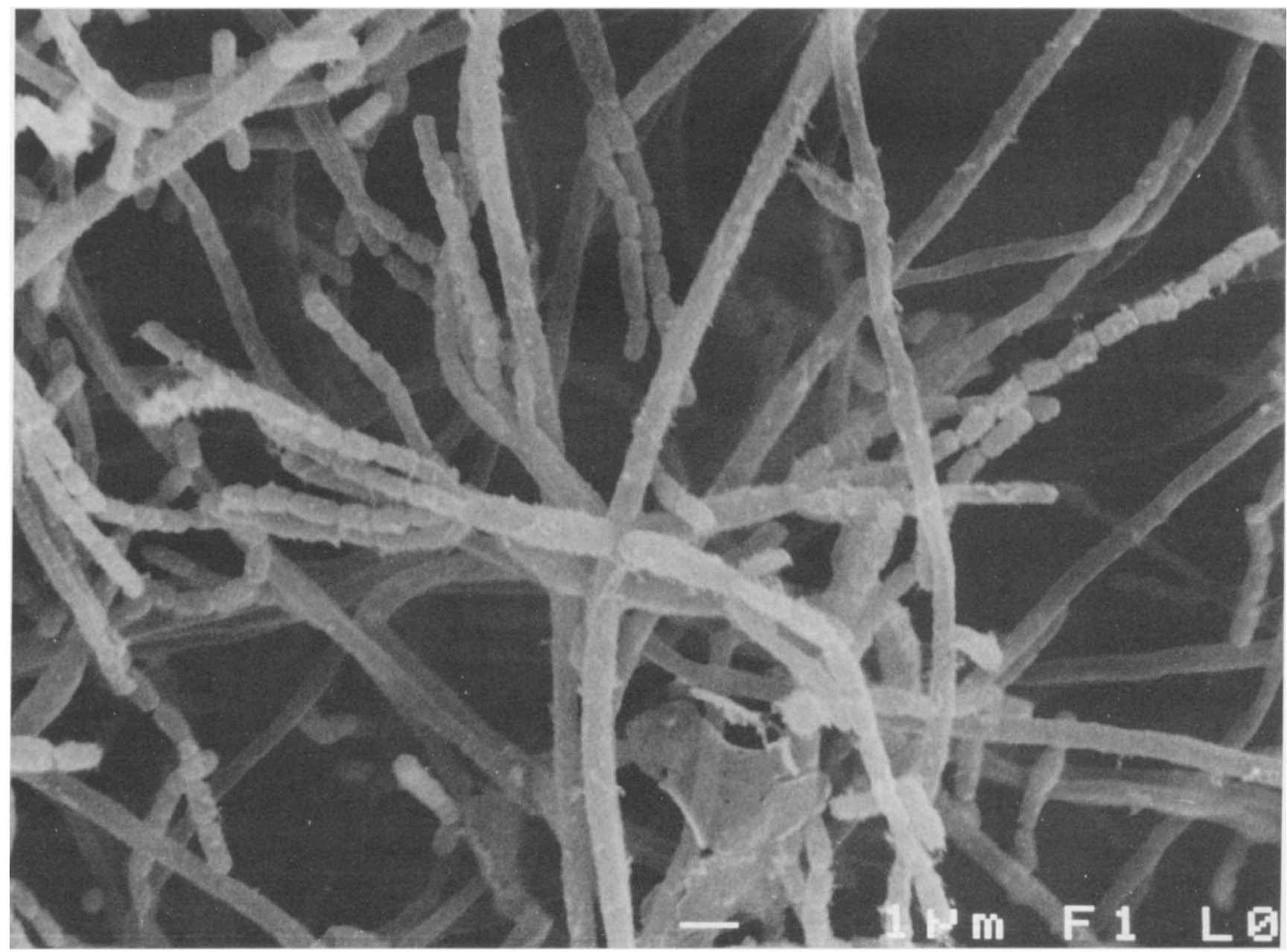

FIG. 2. Scanning electron micrograph of a 14-day culture of $S$. stramineus NRRL $12292^{\mathrm{T}}$ on yeast extract-malt extract agar. Note the umbels of smooth-surface ovoid spores borne on verticils on the aerial mycelia. Bar $=1 \mu \mathrm{m}$ 
TABLE 3. Differential physiological properties of verticillate streptomycetes

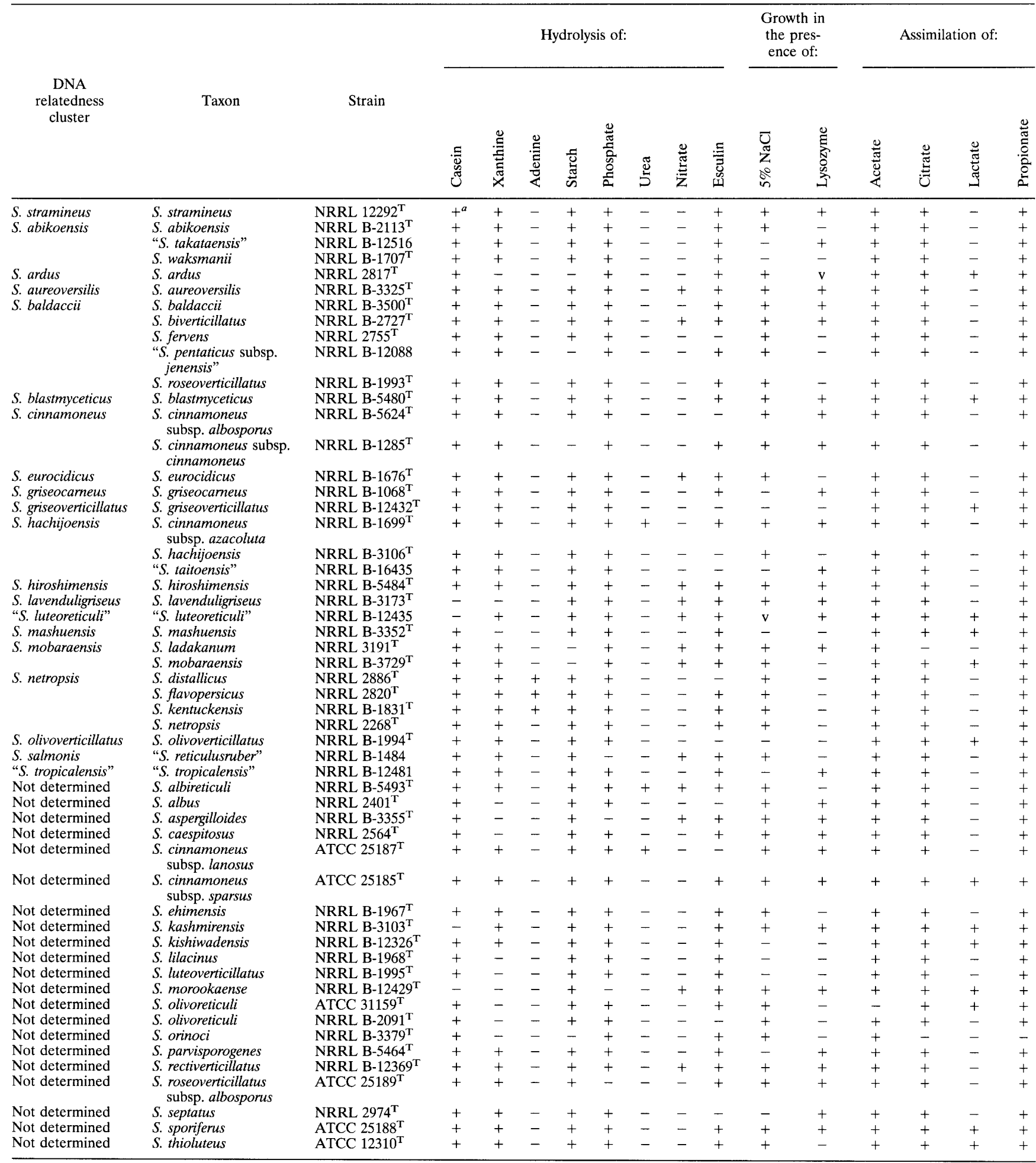

${ }^{a}+$, positive; - , negative; $\mathrm{v}$, variable.

yellow color and cottony nature of the aerial mycelia and spore mass of this strain on most media are particularly distinctive.

Strain NRRL $12292^{\mathrm{T}}$ was found to contain the $\mathrm{L}$ isomer of diaminopimelic acid, which is typical of members of the family
Streptomycetaceae. The predominant fatty acids found in wholecell methanolysates were $15: 0$ anteiso (47\%), $16: 0$ iso (15\%), and $14: 0$ iso $(11 \%)$, which is also typical of streptomycetes.

Physiology. The differential physiological properties of Streptomyces sp. strain NRRL $12292^{\mathrm{T}}$ and the other strains used in 
TABLE 3-Continued

\begin{tabular}{|c|c|c|c|c|c|c|c|c|c|c|c|c|c|c|c|c|c|c|c|c|c|c|c|c|c|c|}
\hline & & & & & & & $\operatorname{cid}$ & $\operatorname{rod}$ & iced & from & & & & & & & & & & & & $\begin{array}{l}\text { rowt } \\
\text { com } \\
\text { ca }\end{array}$ & & $\begin{array}{l}\text { lowi } \\
\text { sole } \\
\text { s: }\end{array}$ & & \\
\hline 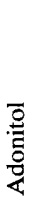 & 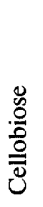 & 畩 & 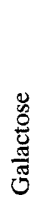 & 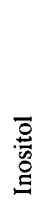 & 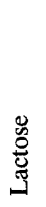 & 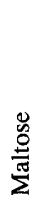 & 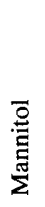 & 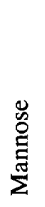 & 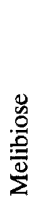 & 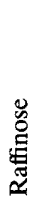 & 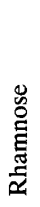 & : & $\begin{array}{l}\overline{0} \\
\text { 율 } \\
\text { \& }\end{array}$ & 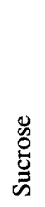 & 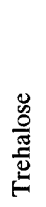 & $\frac{\ddot{g}}{\grave{x}}$ & 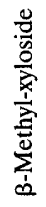 & 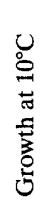 & 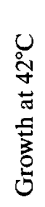 & 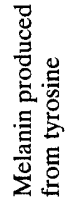 & 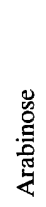 & 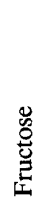 & 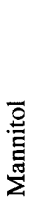 & 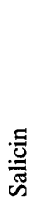 & $\frac{\ddot{y}}{\frac{0}{2}}$ & $\begin{array}{l}\text { Aerial mycelium } \\
\text { color }\end{array}$ \\
\hline+ & - & + & - & + & - & + & + & + & - & - & - & - & + & - & + & - & - & + & + & - & - & + & + & - & - & Yellow \\
\hline - & - & + & + & + & - & + & - & + & - & - & - & - & - & + & + & - & - & - & + & + & - & + & - & - & - & Cinnamon \\
\hline - & - & + & - & + & - & + & + & + & - & - & - & - & - & - & - & - & - & + & + & + & - & + & + & - & - & Off-white \\
\hline - & - & + & - & + & - & + & + & + & - & - & - & - & - & + & - & - & - & - & + & + & - & + & - & - & - & White \\
\hline - & - & + & + & + & - & + & - & + & $\mathrm{v}$ & - & - & - & - & $\mathrm{v}$ & + & - & - & + & + & + & - & + & - & - & - & Light gray \\
\hline - & - & - & - & + & - & + & - & + & - & - & - & - & - & - & + & - & - & + & + & + & - & - & - & - & - & Pink-beige \\
\hline - & - & - & - & + & - & + & - & + & - & - & - & - & - & - & + & - & - & + & + & + & - & - & - & - & - & Cinnamon pink \\
\hline- & - & + & - & + & - & + & - & + & - & - & - & - & - & - & + & - & - & + & + & + & - & + & - & - & - & Bright fluorescent pink \\
\hline - & - & - & - & + & - & + & - & + & - & - & - & - & - & + & + & - & - & + & + & + & + & + & - & - & + & Off-white \\
\hline - & - & + & - & + & - & + & - & + & - & - & - & - & - & - & + & - & - & - & - & + & - & - & - & - & - & Light cinnamon \\
\hline- & - & + & - & + & - & + & - & + & - & - & - & - & - & + & + & - & - & + & + & + & - & + & - & - & - & Pinkish brown \\
\hline - & - & + & + & + & - & + & - & + & - & - & - & - & - & - & + & - & - & + & - & + & - & + & - & - & - & Bald \\
\hline - & - & + & - & + & - & + & - & + & - & - & - & - & - & + & + & - & - & - & + & - & - & - & - & - & - & Cinnamon \\
\hline- & - & - & - & + & - & + & - & + & - & - & - & - & - & - & + & - & - & - & + & + & - & + & - & - & - & Cinnamon \\
\hline - & - & - & - & + & - & + & - & + & - & - & - & - & - & - & + & - & - & + & - & + & - & + & - & - & - & Yellow \\
\hline - & - & - & - & + & - & + & - & + & - & - & - & - & - & - & + & - & - & + & + & + & - & + & - & - & - & Bald \\
\hline - & - & - & + & + & - & + & - & + & - & - & - & - & - & - & + & - & - & + & + & - & - & + & - & - & - & Cinnamon \\
\hline - & - & - & - & + & - & + & - & + & - & - & - & - & - & - & + & - & - & + & + & - & - & - & - & - & - & Light khaki tan \\
\hline - & - & - & - & + & - & + & - & + & - & - & - & - & - & - & + & - & - & + & + & - & - & + & - & - & - & Medium beige \\
\hline - & - & - & - & + & - & + & - & + & - & - & - & - & - & - & + & - & - & + & + & - & - & + & - & - & - & Beige \\
\hline - & - & - & - & t & - & + & - & + & - & - & - & - & - & - & + & - & - & + & + & + & - & + & + & - & - & Pinkish brown \\
\hline - & - & + & + & + & - & + & - & + & + & + & - & - & - & - & + & - & - & + & - & - & - & + & - & - & - & Gray \\
\hline - & - & - & - & + & - & + & - & + & - & - & - & + & - & - & - & - & - & + & + & - & + & + & - & - & - & Gray \\
\hline - & - & + & - & + & - & + & - & + & - & - & - & - & - & + & + & - & - & - & + & + & - & + & - & + & - & Sparse white \\
\hline - & - & - & - & + & - & + & - & + & - & - & - & - & - & + & + & - & - & + & + & - & - & + & - & - & - & Bald \\
\hline - & + & - & - & + & - & + & - & + & - & - & - & - & - & + & + & - & - & - & + & + & - & + & - & - & - & Mouse gray \\
\hline - & - & - & - & + & - & + & - & + & - & - & - & - & - & + & - & - & - & - & + & - & - & + & - & - & - & Gold \\
\hline - & - & + & - & + & - & + & - & + & - & - & - & - & - & + & + & - & - & + & + & - & - & + & - & - & - & Dusty pink \\
\hline - & - & - & - & + & - & + & - & + & - & - & - & - & - & + & + & - & - & t & + & - & - & + & - & - & - & Light beige \\
\hline - & - & + & - & + & - & + & - & + & - & - & - & - & - & - & + & - & - & + & + & - & - & + & - & - & - & Greenish gray-cinnamon \\
\hline - & - & - & + & + & - & + & - & + & - & - & - & - & - & - & + & - & - & + & + & - & - & + & - & - & - & Sparse white \\
\hline - & - & - & - & + & - & + & - & + & - & - & - & - & - & - & + & - & - & + & + & + & - & - & - & - & - & Green-gray \\
\hline - & - & - & - & + & - & + & - & + & - & + & + & - & - & - & + & - & - & + & + & + & - & - & - & - & - & Off-white \\
\hline - & - & - & - & + & - & + & - & t & - & - & - & - & - & + & + & - & - & + & + & + & - & + & - & - & - & Light yellowish white \\
\hline - & - & + & - & - & - & + & - & + & - & - & - & - & - & - & - & - & - & - & + & - & - & + & - & - & - & Pinkish brown \\
\hline - & - & + & + & + & + & + & + & + & + & + & - & + & - & + & + & - & - & + & + & - & - & + & + & - & - & Gray \\
\hline - & - & + & + & + & - & + & - & + & - & - & - & - & - & - & + & - & - & + & + & + & - & + & - & - & - & Off-white \\
\hline - & - & - & - & + & - & + & - & + & - & - & - & - & - & + & + & - & - & - & + & - & - & + & - & - & - & Cinnamon \\
\hline - & - & - & - & + & - & + & - & + & - & - & - & - & - & - & + & - & - & - & + & - & - & + & - & - & - & \\
\hline - & - & + & - & + & - & + & + & + & - & - & - & - & - & - & + & - & - & + & + & + & - & + & + & - & + & Grayish pink \\
\hline+ & - & - & - & + & - & + & - & + & - & - & - & - & - & - & + & - & - & - & + & + & - & - & - & - & - & Gray-cinnamon \\
\hline 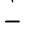 & - & + & - & + & - & + & - & + & - & - & - & - & - & + & + & - & - & - & + & + & - & + & - & + & - & $\begin{array}{l}\text { Pink-beige } \\
\text { Pais }\end{array}$ \\
\hline - & - & - & - & + & - & + & - & + & - & - & - & - & - & + & + & - & - & + & + & + & - & + & - & - & - & Cinnamon \\
\hline 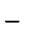 & - & + & + & + & - & + & - & + & - & - & - & - & - & + & + & - & - & + & + & + & - & + & - & - & - & Sparse white \\
\hline - & - & + & + & + & + & + & + & + & + & + & - & + & + & - & + & + & + & + & + & - & - & + & + & - & - & Gray \\
\hline- & - & - & + & + & - & + & - & + & - & - & - & + & - & + & + & - & - & + & + & + & - & + & - & - & - & Gray-white \\
\hline & - & - & + & + & - & + & - & + & - & - & - & - & - & - & + & - & - & + & - & + & - & + & - & - & - & Light beige \\
\hline L & - & - & + & + & + & - & - & - & - & - & - & - & - & - & + & - & - & - & + & - & - & + & - & - & - & Light beige \\
\hline & - & + & + & + & - & + & - & + & - & - & - & - & - & + & + & - & - & + & + & + & - & + & - & - & - & Beige \\
\hline - & - & + & - & + & - & + & - & + & - & - & - & - & - & + & + & - & - & + & + & + & - & + & - & - & - & Pink-beige \\
\hline - & - & - & - & + & - & + & - & + & - & - & - & - & - & + & + & - & - & + & + & + & - & + & - & - & - & Pink-red \\
\hline- & _- & - & - & + & - & + & - & + & - & - & - & - & - & + & + & - & - & + & + & + & - & t & - & - & + & Gray-beige \\
\hline & - & - & - & + & - & + & - & + & - & - & - & - & - & - & + & - & - & - & + & - & - & - & - & - & - & Bald \\
\hline & - & - & - & + & - & + & - & + & - & - & - & + & - & - & - & - & - & - & + & - & - & + & - & - & - & Beige \\
\hline
\end{tabular}

this study are shown in Table 3 . Strain NRRL $12292^{\mathrm{T}}$ is physiologically distinct from the other verticillate strains studied.

DNA relatedness. The levels of DNA relatedness of Streptomyces sp. strain NRRL $12292^{\mathrm{T}}$ to the other verticillate strains included in this study are shown in Table 4. Figure 3 is a graphic representation of the relatedness of strain NRRL $12292^{\mathrm{T}}$ to the other strains after average linkage clustering was performed. Figure 3 shows that Streptomyces sp. species NRRL $12292^{\mathrm{T}}$ clusters separately from all of the other taxa studied.

\section{DISCUSSION}

The morphological properties of strain NRRL $12292^{\mathrm{T}}$ (i.e., production of spore-bearing umbels on verticils formed on the 
TABLE 4. Levels of DNA relatedness between NRRL $12292^{\mathrm{T}}$ and other verticillate Streptomyces strains

\begin{tabular}{|c|c|c|c|}
\hline $\begin{array}{c}\text { DNA } \\
\text { relatedness } \\
\text { cluster }^{a}\end{array}$ & Taxon & Strain & $\begin{array}{c}\% \text { DNA } \\
\text { relatednes } \\
\text { to NRRL } \\
12292^{\mathrm{T}}\end{array}$ \\
\hline \multirow[t]{4}{*}{ S. abikoensis } & S. abikoensis & NRRL B-2113 ${ }^{\mathrm{T}}$ & 8 \\
\hline & "S. rubroverticillatus" & NRRL B-16433 & 16 \\
\hline & "S. takataensis" & NRRL B-12516 & 0 \\
\hline & S. waksmanii & NRRL B- $1707^{\mathrm{T}}$ & 11 \\
\hline S. ardus & S. ardus & NRRL $2817^{\mathrm{T}}$ & 13 \\
\hline S. aureoversilis & S. aureoversilis & NRRL, B- $3325^{\mathrm{T}}$ & 18 \\
\hline \multirow[t]{5}{*}{ S. baldaccii } & S. baldaccii & NRRL B-3500 ${ }^{\mathbf{T}}$ & 0 \\
\hline & S. biverticillatus & NRRL ISP-5272 & 29 \\
\hline & S. fervens & NRRL $2755^{\mathrm{T}}$ & 0 \\
\hline & $\begin{array}{l}\text { "S. pentaticus subsp. } \\
\text { jenensis" }\end{array}$ & NRRL B-12088 & 0 \\
\hline & S. roseoverticillatus & NRRL B-1993 ${ }^{\mathrm{T}}$ & 0 \\
\hline S. blastmyceticus & S. blastmyceticus & NRRL B- $5480^{\mathrm{T}}$ & 31 \\
\hline \multirow[t]{2}{*}{ S. cinnamoneus } & $\begin{array}{l}\text { S. cinnamoneus subsp. } \\
\text { albosporeus }\end{array}$ & NRRL B-5624 ${ }^{\mathrm{T}}$ & 2 \\
\hline & $\begin{array}{l}\text { S. cinnamoneus subsp. } \\
\text { cinnamoneus }\end{array}$ & NRRL B-1285 ${ }^{\mathrm{T}}$ & 12 \\
\hline S. eurocidicus & S. eurocidicus & NRRL B-1676 & 0 \\
\hline S. griseocarneus & S. griseocarneus & NRRL B-1068 ${ }^{\mathrm{T}}$ & 3 \\
\hline S. griseoverticillatus & S. griseoverticillatus & NRRL B-12432 ${ }^{\mathrm{T}}$ & 0 \\
\hline \multirow[t]{3}{*}{ S. hachijoensis } & $\begin{array}{l}\text { S. cinnamoneus subsp. } \\
\text { azacoluta }\end{array}$ & NRRL B-1699 & 0 \\
\hline & S. hachijoensis & NRRL B-3106 ${ }^{\mathrm{T}}$ & 2 \\
\hline & "S. taitoensis" & NRRL B-16435 & 1 \\
\hline S. hiroshimensis & S. hiroshimensis & NRRL B-5484 ${ }^{\mathrm{T}}$ & 18 \\
\hline S. lavendulogriseus & S. lavendulogriseus & NRRL B-3173 & 12 \\
\hline S. luteoreticuli & "S. luteoreticuli" & NRRL B-12435 & 0 \\
\hline S. mashuensis & S. mashuensis & NRRL B-8164 ${ }^{\mathrm{T}}$ & 0 \\
\hline \multirow[t]{2}{*}{ S. mobaraensis } & S. ladakanum & NRRL $3191^{\mathbf{T}}$ & 8 \\
\hline & S. mobaraensis & NRRL B-3729 ${ }^{\mathrm{T}}$ & 3 \\
\hline \multirow[t]{4}{*}{ S. netropsis } & S. distallicus & NRRL $2886^{\mathrm{T}}$ & 26 \\
\hline & S. flavopersicus & NRRL $2820^{\mathrm{T}}$ & 9 \\
\hline & S. kentuckensis & NRRL B-1831 ${ }^{\mathrm{T}}$ & 14 \\
\hline & S. netropsis & NRRL $2268^{\mathbf{T}}$ & 11 \\
\hline S. olivoverticillatus & S. olivoverticillatus & NRRL B-1994 ${ }^{\mathrm{T}}$ & 0 \\
\hline \multirow[t]{2}{*}{ S. salmonis } & "S. reticulusruber" & NRRL B-1484 & 20 \\
\hline & S. salmonis & NRRL B-1472 & 16 \\
\hline S. tropicalensis & "S. tropicalensis" & NRRL B-12481 & 0 \\
\hline
\end{tabular}

a DNA relatedness clusters described by Labeda (5).

aerial mycelia), as shown in Fig. 1 and 2, clearly place this strain with the verticillate streptomycetes, which were formerly classified in the genus Streptoverticillium. The unique straw yellow color of the aerial mycelia and spores of this strain (Table 2) distinguishes it from all other verticillate species; Streptomyces albireticuli and Streptomyces eurocidicus are the only other species with aerial mycelia and/or spores that are yellow. As Table 3 shows, strain NRRL $12292^{\mathrm{T}}$ differs physiologically from the previously described verticillate taxa, including $S$. albireticuli and $S$. eurocidicus, which supports designation of this organism as a member of a new species. The lack of DNA relatedness between strain NRRL $12292^{\mathrm{T}}$ and 34 representative verticillate streptomycetes, as shown by the data in Table 4 and as shown graphically after average linkage clustering in the dendrogram in Fig. 3, confirms that this strain represents a new species, for which the name Streptomyces stramineus is proposed because of the color of the aerial mycelium and spores. A formal description of the species is given below.

Description of Streptomyces stramineus sp. nov. Streptomyces stramineus (stra.min'e.us. L. adj. stramineus, straw colored, re- ferring to the color of the aerial mycelium and spore mass). The substrate mycelium is yellow to yellowish brown. Aerial mycelium forms verticils on which umbels consisting of straight chains of ovoid, smooth-surface, straw yellow spores ( 0.5 by 1.0 $\mu \mathrm{m})$ are produced. Soluble pigments that range from yellowish to shades of brown are produced on several media, such as yeast extract-malt extract agar. Casein, esculin, gelatin, hypoxanthine, starch, tyrosine, and xanthine are hydrolyzed; adenine and urea are not hydrolyzed. Phosphatase is produced; nitrate is not reduced. Melanin pigments are not produced from tyrosine. Growth occurs in the presence of glycerol, $5 \%$ $\mathrm{NaCl}$, and lysozyme; no growth occurs in the presence of salicylate. Acetate, citrate, malate, propionate, pyruvate, and succinate are assimilated; benzoate, lactate, mucate, oxalate, and tartrate are not assimilated. Acid is produced from adonitol, dextrin, fructose, glucose, glycerol, inositol, maltose, mannitol, mannose, sorbitol, and trehalose; no acid is produced from arabinose, cellobiose, dulcitol, erythritol, galactose, melibiose, $\alpha$-methyl-D-glucoside, raffinose, rhamnose, salicin, sucrose, xylose, or $\beta$-methyl-D-xyloside. Growth occurs on fruc-

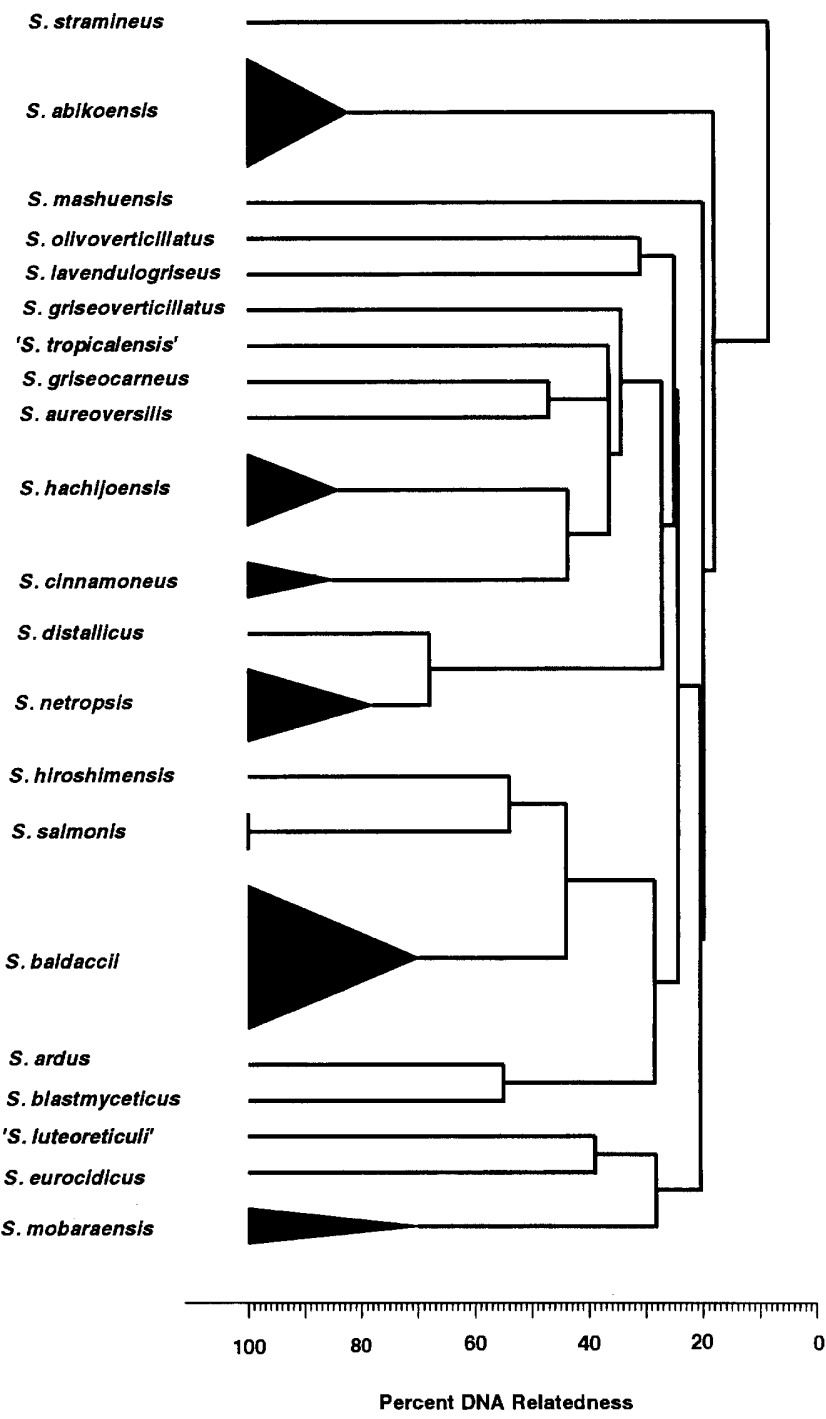

FIG. 3. Dendrogram based on average linkage clustering of levels of DNA relatedness among verticillate Streptomyces species, including $S$. stramineus. S. stramineus is distinct from the other taxa studied. 
tose, glucose, inositol, and mannitol as the sole carbon source but not on galactose, raffinose, rhamnose, salicin, or xylose. Growth occurs at 10 and $42^{\circ} \mathrm{C}$, but not $52^{\circ} \mathrm{C}$. The $\mathrm{G}+\mathrm{C}$ content of the DNA is $75 \mathrm{~mol} \%$ (as determined by the thermal denaturation method). Isolated from a grassland soil sample from South Dakota. Produces the antibiotics LL-BO1208 $\alpha$ and LLBO1208ß. The type strain of S. stramineus is NRRL 12292.

\section{ACKNOWLEDGMENTS}

The able technical assistance of F. L. Baker in preparing the scanning electron micrographs and the assistance of E. N. Hoekstra in isolating and purifying DNA and in performing many of the DNA hybridizations are gratefully acknowledged.

\section{REFERENCES}

1. Becker, B., M. P. Lechevalier, R. E. Gordon, and H. A. Lechevalier. 1964 Rapid differentiation between Nocardia and Streptomyces by paper chromatography of whole-cell hydrolysates. Appl. Microbiol. 12:421-423.

2. Gordon, R. E., D. A. Barnett, J. E. Handerhan, and C. H.-N. Pang. 1974. Nocardia coeliaca, Nocardia autotrophica, and the nocardin strain. Int. J. Syst. Bacteriol. 24:54-63.

3. Kurtzman, C. P., M. J. Smiley, C. J. Johnson, L. J. Wickerham, and G. B. Fuson. 1980. Two new and closely related heterothallic species, Pichia amylophila and Pichia mississippiensis: characterization by hybridization and deoxyribonucleic acid reassociation. Int. J. Syst. Bacteriol. 30:208-216.

4. Kurup, P. V., and J. A. Schmitt. 1973. Numerical taxonomy of Nocardia. Can. J. Microbiol. 19:1035-1048.

5. Labeda, D. P. 1996. DNA relatedness among verticil-forming Streptomyces species (formerly Streptoverticillium species). Int. J. Syst. Bacteriol. 46:699 703.

6. Maniatis, T., E. F. Fritsch, and J. Sambrook. 1982. Molecular cloning: a laboratory manual. Cold Spring Harbor Laboratory, Cold Spring Harbor, N.Y.

7. Marmur, J. 1961. A procedure for the isolation of deoxyribonucleic acid from micro-organisms. J. Mol. Biol. 3:208-218.

8. Marmur, J., and P. Doty. 1962. Determination of the base composition of deoxyribonucleic acid from its thermal denaturation temperature. J. Mol Biol. 5:109-118.

9. Miller, L., and T. Berger. 1985. Bacterial identification by gas chromatography of whole cell fatty acids. Hewlett-Packard application note 228-41. Hewlett-Packard Co., Avondale, Pa.

10. Millonig, G. 1961. Advantages of a phosphate buffer for osmium solutions in fixation. J. Appl. Physiol. 32:1637-1640.

11. Pridham, T. G., and D. Gottlieb. 1948. The utilization of carbon compounds by some Actinomycetales as an aid for species determination. J. Bacteriol. 56:107-114.

12. Pridham, T. G., and A. J. Lyons. 1980. Methodologies for Actinomycetales with special reference to streptomycetes and streptoverticillia, p. 153-224. In A. Dietz and D. W. Thayer (ed.), Actinomycete taxonomy. Special Publication no. 6. Society for Industrial Microbiology, Arlington, Va.

13. Seidler, R. J., M. D. Knittel, and C. Brown. 1975. Potential pathogens in the environment: cultural reactions and nucleic acid studies on Klebsiella pneumoniae from clinical and environmental sources. Appl. Microbiol. 29:819825

14. Seidler, R. J., and M. Mandel. 1971. Quantitative aspects of deoxyribonucleic acid renaturation: base composition, state of chromosome replication, and polynucleotide homologies. J. Bacteriol. 106:608-614.

15. Shirling, E. B., and D. Gottlieb. 1966. Methods for characterization of Streptomyces species. Int. J. Syst. Bacteriol. 16:313-340.

16. Staneck, J. L., and G. D. Roberts. 1974. Simplified approach to identification of aerobic actinomycetes by thin-layer chromatography. Appl. Microbiol. 28:226-231.

17. Witt, D., and E. Stackebrandt. 1990 . Unification of the genera Streptoverticillium and Streptomyces, and emendment of Streptomyces Waksman and Henrici 1943, 339 AL . Syst. Appl. Microbiol. 13:361-371. 